\title{
P03-7-5 Poster session
}

\section{Anti-carcinogenic effects of arctigenin on human breast cancer cells}

\author{
Kyung-Soo Nam, Thressi Maxwell, Kyu-Shik Lee, Soyoung Kim
}

Department of Pharmacology, School of Medcine, Dongguk University, Korea

\section{Background}

Arctigenin is a biologically active plant lignan extracted from Arctium lappa that is consumed worldwide for its several health benefits. However, it might pose a problem for estrogen receptor (ER)-positive human breast cancer patients since several studies show that arctigenin is a phytoestrogen that could exert a proliferative effect by binding to the ER. Therefore, we studied the effect of arctigenin in MCF-7 ER-positive and MDA-MB-231 triple-negative human breast cancer cells.

Methods and Results

SRB demonstrated that arctigenin inhibited the cell viability of MCF-7 cells, and colony forming assay confirmed that this effect was cytotoxic rather than cytostatic. Western blot analysis indicated that arctigenin induced DNA damage without cell cycle arrest, apoptosis, or necroptosis. The increase in lipidated LC3 indicated that arctigenin caused autophagyinduced cell death, which was triggered by an inhibition of mTOR pathway and activation of AMPK. Arctigenin downregulated ERa and its downstream signaling, which was a consequence of the inhibition of mTOR pathway. SRB assay showed that arctigenin did not interfere with the sensitivity of the cells to tamoxifen, anti-cancer drug commonly used in ER-positive breast cancer treatment, but instead exerted a synergistic effect. Furthermore, wound-healing assay and invasion assay demonstrated the antimetastatic effects of arctigenin. RT-PCR, zymography and western blotting revealed that arctigenin exerted its effects by inhibiting MMP9 via the Akt/NF-kB and MAPK/AP-1 signaling pathways. The antimetastatic effects were observed in both ERa positive MCF-7 and triple-negative MDA-MB-231 human breast cancer cells.

Conclusion

Arctigenin was cytotoxic to ER-positive human breast cancer cells, via inhibition of mTOR pathway resulting in autophagy-induced cell death and DNA damage, as well as down regulation of ERa and its downstream signaling. Arctigenin also inhibited cell migration and invasion of human breast cancer cells, irrespective of ERa expression, by inhibiting crucial tumorigenic signaling pathways. Hence, arctigenin is not only safe for consumption by ER-positive breast cancer patients it may also be a natural anti-breast cancer supplement.

[This research was supported by Basic Science Research Program through the National Research Foundation of Korea

(NRF) funded by the Ministry of Education (2015R1D1A01058841)] 